\title{
SAJSM QUESTIONS MARCH 2012
}

\section{CPD questionnaires must be completed online via www.cpdjournals.co.za. \\ After submission you can check the answers and print your certificate.}

\section{True (A) or false (B):}

All studies of fatigue clearly show that it can be attributed to high blood lactate levels.

\section{True (A) or false (B):}

The reason fatigue can be studied easily is because there is a clear concise definition for it.

\section{True (A) or false (B):}

Over a century ago, Mosso wrote that 'fatigue of the brain reduces the strength of the muscles'.

\section{True (A) or false (B):}

Over the years, research has shown that lactic acid is at the origin of all fatigue.

\section{True (A) or false (B):}

A feed-forward mechanism for explaining fatigue attempts to encompass events in which fatigue exists without exhaustion.

\section{True (A) or false (B):}

Following the UN Global Summit on non-communicable diseases, the World Health Organization produced a discussion paper that emphasised the need for increased physical activity.

\section{True (A) or false (B):}

Physical activity is among the top 10 targets of the World Health Organization.

8. True (A) or false (B):

The other targets of the World Health Organization are cardiovascular disease and diabetes, an overall reduction in blood pressure and obesity, reduced smoking, alcohol and dietary salt intake, increased screening for cervical cancer and the elimination of trans-fats from the food supply.

\section{True (A) or false (B):}

Chronic, non-communicable diseases account for more than two-thirds of global mortality, at least $50 \%$ of which is preventable on the basis of modifiable lifestyle behaviours.

10. True (A) or false (B):

In a recent editorial for the British Journal of Sports Medicine, Dr Robert Sallis, Chairman of the Exercise is Medicine Advisory Board, argued that it is as important for physicians to ask a patient about their exercise habits, as it is to measure their blood pressure.

\section{True (A) or false (B):}

About 17\% of the young cricketers surveyed in KwaZuluNatal had continuous musculoskeletal pain.

\section{True (A) or false (B):}

Young cricket players who do not perform sufficient amounts of strength and flexibility training are more predisposed to musculoskeletal pain or injury.

13. True (A) or false (B):

Studies on adult cricket players show that batsmen and wicket-keepers have the highest risk of injury.

14. True (A) or false (B):

Most injuries of adult cricket players occur during matches.

\section{True (A) or false (B):}

The injuries of young cricket players occur in similar proportions between 1-day matches and practices.

\section{True (A) or false (B):}

When compared with the injuries of adult cricket players, young cricketers had a greater risk of injury to their backs and trunks.

\section{True (A) or false (B):}

Prolonged endurance exercise causes muscle damage that initiates an inflammatory response and subsequent muscle remodelling.

18. True (A) or false (B):

The physiological stress after trail running is significantly greater than that which occurs after road running for similar duration.

\section{True (A) or false (B):}

A survey of divers showed that only a few of them had ever experienced symptoms of decompression illness.

\section{True (A) or false (B):}

There is no evidence to suggest that professional divers with high exposure to decompression stress have any resulting intellectual decline.

\section{INSTRUCTIONS}

